\title{
Pediatric Cleft Palate Patients Show a 3- to 5-fold Increase in Cumulative Radiation Exposure from Dental Radiology Compared with an Age- and Gender- matched Population: A Retrospective Cohort Study
}

Reinhilde Jacobs ${ }^{1,2}$, Ruben Pauwels ${ }^{1,3}$, William C Scarfe ${ }^{4}$, Carl De Cock ${ }^{1}$, Karl Dula ${ }^{5}$, Guy Willems ${ }^{6}$, An Verdonck ${ }^{6}$, Constantinus Politis ${ }^{1}$

IOMFS IMPATH research group, Department of Imaging and Pathology, Faculty of Medicine, University of Leuven and Oral \& Maxillofacial Surgery, University Hospitals Leuven, Leuven, Belgium 2Department of Dental Medicine, Karolinska Institutet, Stockholm, Sweden

Radiology and Imaging Science, Department of Surgery and Hospital Dentistry, University of Louisville School of Dentistry, Louisville, Kentucky, USA

Department of Radiology, Faculty of Dentistry, Chulalongkorn University, Bangkok, Thailand ${ }^{5}$ Department of Oral Surgery and Stomatology, School of Dental Medicine, University of Bern, Bern, Switzerland.

${ }^{6}$ Orthodontics, Department of Oral Health Sciences, Faculty of Medicine, University of Leuven

Running title: Pediatric Cumulative Dental Radiation Exposure

Address for correspondence: Reinhilde Jacobs, OMFS-IMPATH research group, Department of Imaging \& Pathology, Faculty of Medicine, University of Leuven, Kapucijnenvoer 33, 3000 Leuven, Leuven, Belgium. E-mail: reinhilde.jacobs@uzleuven.be Telephone: $+32-16-332452$

Conflict of interest: none

Word count abstract: 230 Word count manuscript: 4952 (incl. references)

Tables: 2 Figures: 7 Supplementary files: 1 


\begin{abstract}
Objective: To compare estimates of pediatric cumulative exposure and lifetime attributable risk (LAR) of radiation-induced cancer from dental radiology between cleft palate (CP) subjects and age- and gender-matched controls (non-CP), with and without orthodontic treatment.
\end{abstract}

Materials and Methods: The radiation exposure frequency of CP subjects and nonCP controls with and without orthodontic treatment was compared for two-dimensional radiography (intra-oral, panoramic and cephalometric radiography), computed tomography (CT) and cone-beam CT (CBCT) using cumulative radiation dose as an estimate. From this dose estimate, the age- and gender-dependent risk for radiationinduced stochastic effects was calculated for each patient group.

Results: CP patients received more radiographic examinations than non-CP controls, with the exception of intra-oral radiographs. The cumulative dose to CP patients was considerably higher (1963 $\mu \mathrm{Sv}$ at the age of 20 years) than non-CP patients with (597 $\mu \mathrm{Sv}$ ) and without (383 $\mu \mathrm{Sv}$ ) orthodontic treatment, primarily due to the higher frequency of CT scanning. Accordingly, CP patients had a three to five times higher LAR than non-CP patients.

Conclusions: This study suggests a significantly higher lifetime radiation exposure to $\mathrm{CP}$ patients than non-CP controls from dental radiographic procedures. Diagnostic benefits from the use of CT and CBCT in children must be justified and appropriate dose optimization strategies implemented.

Clinical relevance: The presents study indicates the need for proper justification and optimization of pediatric exposures in dentistry, with a special focus on high-risk groups.

Keywords: Cleft Palate, Pediatric Dentistry, Radiation protection, Radiation dosimetry, Radiation-Induced Cancer 


\section{Introduction}

Radiography is an essential diagnostic modality in dental practice. Dental imaging continues to be the most frequent radiographic diagnostic procedure in medicine, comprising $32 \%$ of all plain radiography procedures in Europe [1]. While individual dental radiographic procedures result in a small effective dose delivered to each patient, the collective population exposure is not trivial because of the frequency of the procedures performed. This may be of concern for children and young adults who are 3 to 5 times more sensitive than adults to radiation-induced carcinogenesis and have many remaining years of life for cancer to develop [2-4]. Recently, concerns have been raised regarding the radiation risks associated with dental diagnostic imaging procedures [5], particularly in association with the occurrence of intracranial meningioma $[6,7]$ and thyroid cancer $[8,9]$.

Many models and types of radiographic units are operating in dental practice providing different types of images. Two-dimensional images are acquired using intraoral, panoramic and cephalometric equipment, whereas three-dimensional imaging is performed using computed tomography (CT) or, more recently, cone-beam CT (CBCT). The latter are often used in pediatric patients requiring corrective or reconstructive surgery, such as cleft palate and orthognathic patients.

Epidemiologic surveys [10] and dosimetric studies using anthropomorphic phantoms [11-18] show that there is tremendous variation in radiation exposure and therefore estimated doses to patients. Variation arises from differences in the radiographic procedure performed, differences between unit models, exposure settings used such as x-ray beam energy, tube output, beam geometry, collimation, the use of thyroid shielding and $\mathrm{x}$-ray sensor [19]. There is a need for both professional and patient education with initiatives such as the "Image Gently in Dentistry" 
campaign, promoting the responsible use of dental and maxillofacial radiographic imaging for children [4]. Image Gently in Dentistry concepts such as "child sizing" radiation exposure has raised awareness about methods to reduce pediatric dose in maxillofacial imaging.

Effective dose $(E)$ is currently the accepted quantitative index of whole-body exposures in terms of detrimental effects, that is, cancer and heritable conditions [20]. However, this metric has a number of limitations including inability to account for gender- or age-specific variations [21]. In oral and maxillofacial radiology, only a few authors have used specific paediatric or adolescent phantoms to calculate effective doses for CBCT procedures [22,23]. Theodorakou et al. calculated average effective doses and mean lifetime attributable risk (LAR) for cancer mortality of five dental CBCT units for representative 10-year old (E, $116 \mu S v$; LAR, 0.00174\%) and 15-year old (E, $79 \mu \mathrm{Sv}$; LAR, 0.00089\%) phantoms [23]. Based on in vivo measurements of skin dose on patients exposed with CBCT, Pauwels et al. reported an average LAR for cancer induction of $0.00060 \%$, with a strong age effect ranging between $0.00098 \%$ for children (age range, $8-11$ years) to $0.00027 \%$ for seniors (age greater than 60 years) [24]. There has been no published report of cumulative radiation-induced cancer risk from $2 \mathrm{D}$ and $3 \mathrm{D}$ comparing pediatric cohorts who receive additional imaging related to their presentation to matched controls.

The purpose of this study is to estimate and compare the cumulative radiation exposure and lifetime attributable risk in a cohort of pediatric cleft palate patients undergoing various dental radiographic procedures with and without orthodontic treatment to age- and gender-matched controls. 


\section{Materials and Methods}

\section{Patient selection}

After institutional review board committee approval (S54932), a retrospective audit was performed of the radiographic records of all patients who presented for dental treatment and received diagnostic dental imaging between 2000 and 2013 (inclusive) at the University Hospitals of Leuven. Only patients at or below 20 years of age at the time of the radiographic exposure were included in the study. All dental radiographic procedures and all exposures were included (including non-diagnostic images). Within this age restricted sample, patients with cleft palate $(\mathrm{CP})$ were identified and age- and gender-matched control cohorts, both with and without orthodontic treatment, were included (Table 1). To ensure that a reliable longitudinal radiographic exposure profile was recorded for all patients in the identified sample at the same institution, recall and treatment records were audited. Patients who had received additional dental imaging by another facility were excluded from the sample. Additional exclusion criteria included patients whose dental records showed one or more long interruptions in treatment (e.g. patients who failed to present for an annual check-up and/or planned dental treatment) or with insufficient information on diagnosis and therapy.

Within the subsample, the age and type of imaging at initial and subsequent dental imaging appointments was obtained, together with the frequency of imaging was determined from review of the chart entries.

To calculate lifetime attributable risk (LAR) for each group stratified for gender, cumulative doses were derived from a summation of individual doses by auditing records detailing the frequency and type of radiographic procedure, knowledge of the type/model of dental radiographic device used and use of recommended and/or reported exposure parameters protocols (exposure time, milliamperage and 
kilovoltage) for each procedure. Five dental radiographic devices were used in our facility between 2000 and 2013 including two panoramic/cephalometric devices (Cranex Tome, Soredex, Tuusula, Finland [2000 to 2008]; Veraviewepocs 2D, Morita, Kyoto, Japan (2008 to 2013]), two CBCT devices (SCANORA 3D, Soredex, Tuusula, Finland; 3D Accuitomo, J. Morita, Kyoto, Japan) and various intraoral devices (MINRAY, Soredex, Tuusula, Finland and Trophy, Carestream, Rochester, NY, USA). In addition, CT scans were obtained from different units of the Somatom series (Siemens, Erlangen, Germany). An overview of the radiographic techniques and corresponding devices included in this study is shown in Appendix A. This table also contains other equipment than that used for the patient sample in this study, if this equipment provided a better match in terms of radiation exposure than published values for the actual equipment in question, or if dose estimates for the equipment used at our facility were not available.

The effective dose was chosen as metric for patient radiation dose as it reflects the overall detriment caused by the radiation exposure to radiosensitive organs and tissues, and because it is the only way to compare exposures from different modalities. Effective doses were determined for two-dimensional radiography (including panoramic, cephalometric and intra-oral imaging), СBCT and multi-detector CT (MDCT) studies (Appendix A) based on available dosimetric literature. For older studies which reported effective dose according to ICRP 60 [20], the effective dose was recalculated using ICRP 103 weighting factors [25] by means of absorbed organ doses, when available. Effective doses from studies measured for paediatric patients were used when available, or corrected/interpolated according to patient age if feasible. For the Veraviewepocs 2D panoramic unit, instead of relying on published effective dose, dose area product (DAP) values were obtained from all paediatric 
patients. These DAP-values were converted to effective dose using previously determined conversion factors [26]. For $\mathrm{CBCT}$ and $\mathrm{CT}$, values in the literature matching most closely with the combination of exposure parameters (e.g. field of view, mAs) used for each patient were used. When possible, the effective dose of CT images was derived from exposure parameters that were saved along with the CT images.

Finally, an estimation of the age-dependent risk for radiation-induced cancer was made for each patient group using previously reported data in the BEIR VII report [27]. In this publication, the overall Lifetime Attributable Risk (LAR) for a $100 \mathrm{mSv}$ dose is provided as a function of gender and age at exposure. Using the effective dose estimations for each patient group at a given age, , the LAR can be estimated for a female, male and gender-averaged patient.

Statistical analysis was performed using Excel 10 (Microsoft Corp., Redmond, WA, USA). Student's t-test was performed to compare the differences in the accumulated radiation dose and the LAR between groups and between genders using a Bonferroni correction with $p \leq 0.017$ and $p \leq 0.05$ used as the level of significance for each dependent variable. 


\section{Results}

Applying the inclusion and exclusion criteria, a total of 504 patients were included, with 102 having CP and 402 non-CP age- and gender-matched controls. Of the non-CP individuals, 231 had orthodontic treatment and 171 had no orthodontic treatment (Table 1).

\section{Age at First Dental Image}

On average, non-CP patients without orthodontic treatment had their first panoramic radiograph taken at the age of 11 years, whereas those with orthodontic treatment underwent panoramic radiography 4 months earlier. For CP patients, initial panoramic radiographic exposure was on average approximately 2 years earlier than controls. For intraoral imaging, non-CP patients received initial exposures at 11 years 6 months irrespective of orthodontic treatment, whereas for $\mathrm{CP}$ patients this imaging was performed 2 months earlier. For cephalometric radiography, non-CP individuals were, on average, imaged more than a year and a half after initial panoramic imaging, with slight differences between those with (12 years 4 months) and without orthodontic treatment (12 years 9 months). For CP individuals cephalometric imaging was performed, on average, 4 years earlier ( 8 years 6 months) than panoramic procedures. Generally, initial imaging was performed earlier for females than males.

\section{Frequency of Dental Imaging}

Figs. 1-5 show the percentage of individuals who received one or more specific imaging procedures according to age group.

For panoramic radiography (Fig. 1), both the percentage of individuals exposed and the frequency of radiographs was higher for CP than for non-CP individuals. The 
most pronounced difference was found in the $6-10$ year age group, $91 \%$ of CP patients received panoramic exposures (average frequency: 2.76 ), whereas $42 \%$ of non-CP patients with orthodontic treatment received, on average, 1.77 exposures, and $32 \%$ of non-CP patients without orthodontics received 1.58 panoramic exposures.

For intraoral radiography (Fig. 2), overall, an increasing percentage of CP and non-CP individuals with orthodontics received imaging from age 6 until age 16, after which the percentage reduced slightly. Interestingly, a significantly higher $(p<0.01)$ percentage of non-CP individuals without orthodontic treatment were exposed compared with the other groups, with these individuals also having the highest frequency of exposure over all age groups of 6 years and older (range, 4.06 to 4.57 ). Both the percentage and frequency (range, 2.00-2.94) of intra-oral radiographs was lowest for CP patients in age groups of 6 years and older.

The majority of cleft palate patients $(58 \%)$ received a cephalometic radiograph between the age of six and ten (Fig. 3), , which is considerable more than children with (13\%) and without (5\%) orthodontic treatment. For ages 11-13 and 14-16, the percentage of cephalometric radiographs was similar for CP and non-CP orthodontic patents, and much higher than corresponding percentages for non-CP patients without orthodontic treatment. The use of cephalometric radiographs declined at a later age, except for CP patients, for whom the percentage remained relatively stable. In most cases, the frequency of cephalometric radiography per age group was 1 ; most notably, this frequency was slightly higher (1.41) for cleft patients aged 6-10 years.

For CT imaging (Fig. 4) overall, the percentage of cleft patients receiving a CT was significantly higher $(p<0.01)$ compared to non-CP patients. For non-CP patients, the percentage of CT scans was lower for patients not undergoing orthodontic 
treatment, except in the 17-19 year age group. Overall, the percentage of CTs trended downward with age, but increased again in the 17-19 age group.

For CBCT (Fig. 5), a significantly higher percentage of CP patients were scanned compared with patients without orthodontic treatment. In the older patient groups (> 13 years), the number of CBCT scans for $\mathrm{CP}$ and non-CP orthodontic patients was similar, yet considerably higher than that of non-CP non-orthodontic patients. Unlike the data for CT, no patients below 6 years underwent CBCT scanning.

\section{Cumulative Radiation Dose}

The cumulative radiation dose throughout childhood for the three patient groups is shown for two-dimensional radiographs (Fig. 6A), for two-dimensional radiographs and CBCT (Fig. 6B) and for all exposures, including CT (Fig. 6C).

\section{$\underline{\text { Two-dimensional radiographs }}$}

Considering the radiation dose per annum from two-dimensional radiographs, for $\mathrm{CP}$ patients, a peak was reached at the age of 9 years with $6 \mu \mathrm{Sv} / \mathrm{year}$, which remained stable at 5-6 $\mu \mathrm{Sv} / \mathrm{year}$ for the remainder of childhood. Then, another peak was seen for CP patients at 18 years for females $(8 \mu \mathrm{Sv} / \mathrm{year})$ and at 19 years for males (10 $\mu \mathrm{Sv} /$ year). Until the age of ten years, almost no difference can be seen between nonCP patients with and without orthodontic treatment. Accumulated radiation doses from two-dimensional radiographs of CP patients were four times $(p<0.017)$ higher than those of the other two groups at the age of $10(20 \mu \mathrm{Sv} v \mathrm{vs} .5 \mu \mathrm{Sv})$. At the age of 15 years, CP patients had a two to three times higher radiation dose than non-CP patients with and without orthodontic treatment. In control subjects with and without orthodontic treatment, an accumulated dose of $20 \mu \mathrm{Sv}$ was reached at 13 and 19 years for patients 
with and without orthodontic treatment, respectively.

At the age of 20 years, an accumulated dose of $67 \mu \mathrm{Sv}$ (females) and $69 \mu \mathrm{Sv}$ (males) for two-dimensional radiographs was reached for CP patients, contributing to $4 \%$ of the total radiation dose received by dental radiographs, CBCT and CT in the maxillofacial area. For non-CP orthodontically treated patients, an exposure of $44 \mu \mathrm{Sv}$ (females) and $49 \mu \mathrm{Sv}$ (males), contributing to $8 \%$ and $7 \%$ of the total dose, was seen at the age of 20 years. The lowest radiation dose was seen for controls without orthodontic treatment at the age of 20 years, equal to $23 \mu \mathrm{Sv}$ for both females and males, corresponding to $7 \%$ and $5 \%$ of the total dose, respectively (Fig. 6A).

\section{$\underline{\text { Two dimensional radiographs and } \mathrm{CBCT}}$}

A considerable increase in the annual and cumulative dose is observed when the dose from CBCT examinations is added (Fig. 6B). Statistically, the accumulated dose at 20 years was significantly higher $(p<0.01)$ for all groups compared with the dose from two-dimensional radiographs only. The strongest increase in absolute dose and dose ratio, compared to the accumulated dose without CBCT (Fig. 6A), was found in the CP group. For this group, at the age of 20 years, an accumulated dose of $206 \mu \mathrm{Sv}$

(females) and $235 \mu \mathrm{Sv}$ (males) was reached; this amounts to $10 \%$ and $13 \%$, respectively, of the total radiation dose in the maxillofacial area. For both other groups, a 2.2-fold (with orthodontic treatment) and almost 5-fold (without orthodontics) lower accumulated dose, at age 20 years, can be seen.

\section{All exposures (incl. CT)}

Considering the cumulative doses from radiographic procedures, including both CBCT and $\mathrm{CT}$, an average cumulative dose to CP patients of $872 \mu \mathrm{Sv}$ was attained at the 
age of 10 years (Fig. 6C). For patients with orthodontic treatment, on average, a radiation dose of $156 \mu \mathrm{Sv}$ (females) and $390 \mu \mathrm{Sv}$ (males) was seen at age 10 years. For patients without orthodontic treatment, corresponding doses were $155 \mu \mathrm{Sv}$ (females) and $245 \mu \mathrm{Sv}$ (males).

An accumulated radiation dose of $1963 \mu \mathrm{S} v$ was reached at the age of 20 years for cleft patients, compared with $383 \mu$ Sv for non-CP non-orthodontic patients and 597 $\mu S v$ for non-CP orthodontic patients.

\section{Lifetime attributable risk}

LAR results are shown in Fig. 7 and Table 2. For CP patients, when including CBCT and excluding $\mathrm{CT}$, the average LAR, expressed as radiation-induced cancer cases per million (pm) at the age of 20 was $2.8 \mathrm{pm}$ for males and $5.0 \mathrm{pm}$ for females $(3.8 \mathrm{pm}$ overall). Including all CT radiographs of the jaw and facial region, the overall LAR at the age of 20 was $40.4 \mathrm{pm}$, being over ten times higher for males $(30.1 \mathrm{pm})$ and females (54.5 pm) than when CT was excluded.

Including CT and CBCT, at 20 years of age, the LAR for CP patients was 3.3 times higher than that of non-CP orthodontic patients, and 5.1 times higher than nonCP non-orthodontic patients. Consequently, the LAR for orthodontic patients was 1.6 times higher than that of non-orthodontic patients. Typically, the risk ratio between $\mathrm{CP}$ and non-CP patients peaked at a young age (5-10 y), after which it remained stable or declined at an older age. 


\section{Discussion}

Based on the cohort of patient populations in this study, we found that CP patients receive a 3- to 5-fold higher exposure due to dental radiological examinations than non-CP patients.. Whereas CP patients underwent a higher number of exposures from all radiographic modalities except intra-oral radiography, the cumulative dose as well as the LAR was mainly determined by CT. This indicates the need to justify and optimize the use of CT for CP patients in particular. CP patients not only received a higher number of CT scans at a young age, but the dose for these CTs tends to be higher as well. This may be due to CT procedures of the temporal bones, which may be acquired because of conditions related to the auditory system. Therefore, in cases where three-dimensional high detail bone imaging is required, the use of CBCT should be considered instead of CT. This could yield diagnostic information at a lower dose than CT, especially if the FOV is collimated to a small region of interest [18]. For the scanning of larger regions, CT examinations can be performed at low-dose protocols due to recent improvements in hardware and the use of iterative reconstruction algorithms [28,29]. However, the choice of volumetric 3-D modality should also be based on the required diagnostic image quality, balancing higher detail of CBCT images with reduced contrast and higher noise [30]. For patients with/without orthodontic treatment, who undergo 3D scanning for indications such as assessment of an impacted tooth, trauma, orthodontic and endodontics, the same considerations apply. Initially, the first CBCT unit at our institution was installed in 2004 and was primarily used for dental implant and endodontic treatment planning. Since then, three additional CBCT units have been installed and their clinical use, particularly for pediatric patients, has increased. The frequency of CBCT imaging found for this sample, which considered the period 2000-2013, does not reflect this increased 
utilization. It is likely that the frequency of CT examinations for these patient groups has steadied or even decreased, along with an increase in the use of low-dose protocols in $\mathrm{CT}$, but that the use of CBCT has increased considerably, leading to a higher collective dose. Due to the recent emergence of CBCT imaging for pediatric applications, dedicated research evaluating the cost-benefit ratio for specific clinical indications is needed.

This study also revealed a difference in exposure between orthodontic and nonorthodontic patients, with the former group receiving a 1.6-fold increase in exposure, on average. Panoramic radiographs are used to support pre-treatment planning and post-treatment evaluation in orthodontics. This modality provides a large amount of information on a single procedure at a relatively low radiation dose, compared to the dose produced by CT or CBCT procedures that cover the same anatomic region. The cumulative number of imaging procedures performed during or after orthodontic treatment, should all be justified on an individual need basis to achieve the optimal treatment goals using a minimal radiation exposure. On that note, it is somewhat concerning to see that orthodontic patients also tend to undergo more CT and CBCT examinations than non-orthodontic patients, as there is still a need for evidence regarding the benefit of these modalities in terms of orthodontic treatment outcome [31].

We found that the LAR for females was higher than for males; this may be for a number of reasons. First, on average, females are often examined and imaged at an earlier age, because of their earlier dental development [32,33]. Secondly, the risk per dose is higher for females, with the difference in risk between genders increasing at younger ages. Pauwels et al. estimated a $40 \%$ average higher LAR for females due to dental CBCT examinations over all ages (incl. adults) [24]. At younger ages, the risk 
per dose is almost twice as high for females than males (Table 2) [27], suggesting a need for more stringent justification and optimization of pediatric exposures. Third, it is possible that because of average head size discrepancies, female doses may be higher than those for males at a given age.

Doses in this study were derived from a multitude of publications, most of which involving direct measurements of organ doses using anthropomorphic phantoms. This may contribute to some degree of uncertainty associated with the dose estimations performed in this study. Therefore, it is recommended that values provided in this report are interpreted with caution. Absolute doses, even when applied at a population level, are highly variable due to effects of exposure parameters, patient anatomy, and measurement methodology. While we attempted to correct for these factors as much as possible (e.g. by recalculating older published value using ICRP 103 weighting factors), uncertainty cannot be avoided. Future epidemiological studies, especially if prospective, should focus on accurate dose estimation, correcting for as many equipment- and patient-specific factors as possible. Another obvious limitation of the effective dose is that it is calculated using a single set of tissue weighting factors; thus, by definition, it is independent of age and gender. However, it is well-known that the radiosensitivity of different organs is highly age- and gender-dependent.

The conversion of dose to risk adds an additional layer of uncertainty. While the aforementioned limitation of effective dose is addressed by the fact that the LAR estimation takes differences in radiosensitivity due to age and gender into account, conversion factors available at the moment are primarily derived from the atomic bomb survivors [34-36], and are prone to uncertainty at doses below $100 \mathrm{mSv}$. The conversion factors rely on the assumption of compliance with the linear non-threshold model and the use of a dose and dose rate effectiveness factor (DDREF), the value 
of which has been under debate, to correct for the lower biological effectiveness at low doses and dose rates. The applicability of the effective dose, which relies on averaged and rounded tissue weighting factors, has also been questioned for non-whole-body exposures [25], but it remains the most conventional metric to express the dose from medical exposures in literature. Whereas cancer risks can also be determined for each individual exposed organ, the LAR derived from the effective dose sufficed in this study in order to differentiate CP and non-CP patients.

In conclusion, the results from this study suggest that radiographs in the maxillofacial area of children, especially females, with cleft palate might result in a significantly increased lifetime attributable risk compared with a control cohort. This is due to an increased cumulative exposure, mainly from additional CT procedures, occurring at a younger age. Further research on larger cohorts and prospective study samples is necessary to verify these results.

\section{Compliance with Ethical Standards}

Conflict of Interest: Author Reinhilde Jacobs declares that she has no conflict of interest. Author William C Scarfe declares that he has no conflict of interest. Author Ruben Pauwels declares that he has no conflict of interest. Author Carl De Cock declares that he has no conflict of interest. Author Karl Dula declares that he has no conflict of interest. Author Guy Willems declares that he has no conflict of interest. Author Constantinus Politis declares that he has no conflict of interest. Author An Verdonck declares that she has no conflict of interest.

Funding: No funding Support

Ethical approval: All procedures performed in studies involving human participants 
were in accordance with the ethical standards of the institutional and/or national research committee and with the 1964 Helsinki declaration and its later amendments or comparable ethical standards. After institutional review board committee approval (S54932), a retrospective audit was performed of the radiographic records of all patients who presented for dental treatment and received diagnostic dental imaging between 2000 and 2013 (inclusive) at the University Hospitals of Leuven.

Informed consent: Regardless of the retrospective nature of this study, informed consent was obtained from all individual participants included in the study . 


\section{References}

1. European Comission (2014) RADIATION PROTECTION $N^{\circ}$ 180: Medical Radiation Exposure of the European Population. Part 1/2: Publications Office of the European Union. Luxembourg:1-181.

2. Brenner D, Elliston C, Hall E, Berdon W (2001) Estimated risks of radiation-induced fatal cancer from pediatric CT. AJR Am J Roentgenol 176:289-296.

3. Miglioretti DL, Johnson E, Williams A, et al (2013) The use of computed tomography in pediatrics and the associated radiation exposure and estimated cancer risk. JAMA Pediatr 167:700-707.

4. White SC, Scarfe WC, Schulze RK, et al (2014) The Image Gently in Dentistry campaign: promotion of responsible use of maxillofacial radiology in dentistry for children. Oral Surg Oral Med Oral Pathol Oral Radiol 118:257-261.

5. Lin MC, Lee CF, Lin CL, et al (2013) Dental diagnostic X-ray exposure and risk of benign and malignant brain tumors. Ann Oncol 24:1675-1679.

6. Longstreth, WT Jr., Phillips LE, Drangsholt M, et al (2004) Dental X-rays and the risk of intracranial meningioma: a population-based case-control study. Cancer 100:1026-1034.

7. Claus EB, Calvocoressi L, Bondy ML, Schildkraut JM, Wiemels JL, Wrensch M (2012) Dental x-rays and risk of meningioma. Cancer 118:4530-4537.

8. Neta G, Rajaraman P, Berrington de Gonzalez A, et al (2013) A prospective study of medical diagnostic radiography and risk of thyroid cancer. Am J Epidemiol 177:800-809.

9. Memon A, Godward S, Williams D, Siddique I, AI-Saleh K (2010) Dental X-rays and the risk of thyroid cancer: a case-control study. Acta Oncol 49:447-453.

10. Conference of Radiation Control Program Directors (CRCPD) (2003) Nationwide Evaluation of X-ray Trends (NEXT) Tabulation and Graphical Summary of 1999 Radiography Survey, CRCPD publication E-03-6

11. Garcia Silva MA, Wolf U, Heinicke F, Gründler K, Visser H, Hirsch E (2008) Effective dosages for recording Veraviewepocs dental panoramic images: analog film, digital, and panoramic scout for CBCT. Oral Surg Oral Med Oral Pathol Oral Radiol Endod 106:571-577.

12. Han GS, Cheng JG, Li G, Ma XC (2013) Shielding effect of thyroid collar for digital panoramic radiography. Dentomaxillofac Radiol 42:20130265.

13. Gavala S, Donta C, Tsiklakis K, Boziari A, Kamenopoulou V, Stamatakis HC (2009) Radiation dose reduction in direct digital panoramic radiography. Eur $\mathrm{J}$ Radiol 71:42-8.

14. Kaeppler G, Dietz K, Reinert S (2007) Possibilities of dose reduction in lateral cephalometric radiographs and its effects on clinical diagnostics. Dentomaxillofac Radiol 36:39-44.

15. Gijbels F, Sanderink G, Wyatt J, Van Dam J, Nowak B, Jacobs R (2004) Radiation doses of indirect and direct digital cephalometric radiography. Br Dent J 197:149152.

16. Ludlow JB, Davies-Ludlow LE, White SC (2008) Patient risk related to common dental radiographic examinations: the impact of 2007 International Commission on Radiological Protection recommendations regarding dose calculation. J Am Dent Assoc 139:1237-1243.

17. Johnson KB, Ludlow JB, Mauriello SM, Platin E (2014) Reducing the risk of intraoral radiographic imaging with collimation and thyroid shielding. Gen Dent 62:34-40. 
18. Pauwels R, Beinsberger J, Collaert B, et al (2012) Effective dose range for dental cone beam computed tomography scanners. Eur J Radiol 81:267-271.

19. Brink JA, Boone JM, Feinstein KA, et al (2012) Reference levels and achievable doses in medical and dental imaging: recommendations for the United States. NCRP Report No. 172.

20. International Commission on Radiological Protection (1991) 1990 Recommendations of the International Commission on Radiological Protection. ICRP Publication 60. Ann Int Comm Radiol Protect 21:1-201.

21. Wall B, Haylock R, Jansen JTM, Hillier MC, Hart D, Shrimpton PC (2011) Radiation Risks from Medical X-ray Examinations as a Function of the Age and Sex of the Patient. Report HPA-CRCE-028. Health Protection Agency, Chilton.

22. Al Najjar A, Colosi D, Dauer LT, et al (2013) Comparison of adult and child radiation equivalent doses from 2 dental cone-beam computed tomography units. Am J Orthod Dentofacial Orthop 143:784-792

23. Theodorakou C, Walker A, Horner K, et al (2012) Estimation of paediatric organ and effective doses from dental cone beam CT using anthropomorphic phantoms. Br J Radiol 85:153-160.

24. Pauwels R, Cockmartin L, Ivanauskaité D, et al (2014) Estimating cancer risk from dental cone-beam CT exposures based on skin dosimetry. Phys Med Biol 59:38773891.

25. International Commission on Radiological Protection (2007) The 2007 Recommendations of the International Commission on Radiological Protection. ICRP Publication 103. Ann ICRP 37:1-332.

26. Looe HK, Eenboom F, Chofor N, et al (2008) Conversion coefficients for the estimation of effective doses in intraoral and panoramic dental radiology from dosearea product values. Radiat Prot Dosimetry 131:365-373.

27. Committee to Assess Health Risks from Exposure to Low Levels of Ionizing Radiation (2006) Health Risks from Exposure to Low Levels of Ionizing Radiation: BEIR VII Phase 2. Washington, DC: The National Academies Press.

28. Widmann G, Bischel A, Stratis A, et al (2016) Ultralow dose dentomaxillofacial CT imaging and iterative reconstruction techniques: variability of Hounsfield units and contrast-to-noise ratio. Br J Radiol 89:20151055.

29. Widmann G, Schullian P, Gassner EM, Hoermann R, Bale R, Puelacher W (2015) Ultralow-dose CT of the craniofacial bone for navigated surgery using adaptive statistical iterative reconstruction and model-based iterative reconstruction: 2D and 3D image quality. AJR Am J Roentgenol 204:563-569.

30. Pauwels R, Beinsberger J, Stamatakis $\mathrm{H}$, et al (2012) Comparison of spatial and contrast resolution for cone-beam computed tomography scanners. Oral Surg Oral Med Oral Pathol Oral Radiol 114:127-35.

31. European Commission. Radiation Protection Publication nr. 172. Cone beam CT for dental and maxillofacial radiology - Evidence based guidelines. Available on https://ec.europa.eu/energy/sites/ener/files/documents/172.pdf

32. Wedl JS, Danias S, Schmelzle R, Friedrich RE (2005) Eruption times of permanent teeth in children and young adolescents in Athens (Greece). Clin Oral Investig 9:131-134.

33. Wedl JS, Schoder V, Blake FAS, Schmelzle R, Friedrich RE (2004) Eruption times of permanent teeth in teenage boys and girls in Izmir (Turkey). J Clin Forensic Med 11:299-302.

34. Pierce DA, Preston DL (2000) Radiation-related cancer risks at low doses among atomic bomb survivors. Radiat Res 154:178-186. 
35. Preston DL, Shimizu Y, Pierce DA, Suyama A, Mabuchi K (2003) Studies of mortality of atomic bomb survivors. Report 13: Solid cancer and noncancer disease mortality: 1950-1997. Radiat Res 160:381-407.

36. Preston DL, Ron E, Tokuoka S, Funamoto S, Nishi N, Soda M, et al (2007) Solid cancer incidence in atomic bomb survivors: 1958-1998 Radiat Res 2007;167:1-64. 


\section{Figure legends}

Figure 1. Percentage of individuals who received one or more panoramic images according to age group.

Figure 2. Percentage of individuals who received one or more intraoral radiographs according to age group.

Figure 3. Percentage of patients who received one or more cephalometric radiographs according to age group.

Figure 4. Percentage of individuals who received one or more CT imaging procedures according to age group.

Figure 5. Percentage of individuals who received one or more CBCT imaging procedures according to age group.

Figure 6. Accumulated radiation dose throughout childhood for (A) two-dimensional radiography, (B) two-dimensional radiography and $\mathrm{CBCT}$ and $(\mathrm{C})$ all maxillofacial radiographic procedures (incl. CT)

Figure 7. Lifetime Attributable Risk due to (A) two-dimensional radiography, (B) twodimensional radiography and $\mathrm{CBCT}$ and $(\mathrm{C})$ all maxillofacial radiographic procedures (incl. CT) 